\title{
A modified Tikhonov regularization method for a class of inverse parabolic problems
}

\author{
Nabil SAOULI and Fairouz ZOUYED
}

\begin{abstract}
This paper deals with the problem of determining an unknown source and an unknown initial condition in a abstract final value parabolic problem. This problem is ill-posed in the sense that the solutions do not depend continuously on the data. To solve the considered problem a modified Tikhonov regularization method is proposed. Using this method regularized solutions are constructed and under boundary conditions assumptions, convergence estimates between the exact solutions and their regularized approximations are obtained. Moreover numerical results are presented to illustrate the accuracy and efficiency of the proposed method.
\end{abstract}

\section{Introduction}

Let $H$ be a separable Hilbert space with the inner product (.,.) and the norm \|. . and let $A: D(A) \subset H \rightarrow H$ be a positive self-adjoint linear operator with a compact resolvent. Consider the following final value problem

$$
\left\{\begin{array}{l}
u_{t}(t)+A u(t)=f, \quad 0<t<T_{2}, \\
u\left(T_{1}\right)=\psi_{1},
\end{array}\right.
$$

Key Words: Parabolic problem, ill-posed problem, inverse problem, modified Tikhonov regularization, error estimate.

2010 Mathematics Subject Classification: Primary 35R30, 47A52; Secondary 35K90, $35 \mathrm{R} 25$.

Received: March, 2019.

Revised: April, 2019.

Accepted: May, 2019. 
where $0<T_{1}<T_{2}$ and $\psi_{1}$ is a given function on $H$. Our purpose is to identify the initial condition $u(0)$ and the unknown source $f$ from the overspecified data

$$
u\left(T_{2}\right)=\psi_{2}, \quad \psi_{2} \in H .
$$

Hence, the inverse problem can be formulated as follows: determine $f$ and $g$ such that

$$
\left\{\begin{array}{l}
u_{t}(t)+A u(t)=f, \quad 0<t<T_{2}, \\
u(0)=g
\end{array}\right.
$$

from the data

$$
\left\{\begin{array}{l}
u\left(T_{1}\right)=\psi_{1}, \\
u\left(T_{2}\right)=\psi_{2} .
\end{array}\right.
$$

As we know, the method of abstract differential equations provides proper guidelines for solving various problem with partial differential equations involved. As an example of (1.2) we introduce the following problem, let $\Omega$ be a bounded domain in the space $\mathbb{R}^{n}$, whose boundary is sufficiently smooth and set $\Omega_{T}=\Omega \times\left[0, T_{2}\right], \Sigma_{T}=\partial \Omega \times\left[0, T_{2}\right]$. Consider the initial boundary value problem for the heat conduction equation given by

$$
\left\{\begin{array}{l}
\frac{\partial u}{\partial t}(x, t)-\Delta u(x, t)=f(x), \quad(x, t) \in \Omega_{T}, \\
u(x, t)=0, \quad(x, t) \in \Sigma_{T} \\
u(x, 0)=g(x), \quad x \in \Omega .
\end{array}\right.
$$

Adopting $H=L^{2}(\Omega)$, the operator $A$ is taken to be $A=\Delta$ with the domain $D(A)=H_{0}^{1}(\Omega) \cap H^{2}(\Omega)$. The direct problem related to the heat equation is to determine the temperature distribution from the knowledge of the initial temperature the source term and the boundary conditions, which generally leads to a well-posed problem. However, it is not always possible to specify the initial temperature or the source term or the both functions in many practical situations. So an inverse problem is raised: we have to determine the $u(x, 0)$ and $f(x)$ from observations at moments $T_{1}$ and $T_{2}$ i.e.;

$$
u\left(x, T_{1}\right)=\psi_{1}(x), \quad u\left(x, T_{2}\right)=\psi_{2}(x), \quad x \in \Omega, 0<T_{1}<T_{2},
$$

where $\psi_{1}$ and $\psi_{2}$ are two given functions on $H$.

The main difficulty in the study of the inverse problem (1.2)-(1.3) (respectively $(1.4)-(1.5)$ ) (as we will see in section 2) is that it is ill-posed i.e., even if a solution exists, it is unstable. The lack of property of stability creates a serious problem if one wants to approximate the solution by numerical methods. 
Thus, special regularization methods that restore the stability with respect to measurements errors are needed.

We point out that although the parabolic equations are very popular and widely studied in the literature of inverse problems for PDEs, such as the backward parabolic equations see $[2,5,9,11,14,16,22]$ and the references therein and the identification of the source term in heat equation see, e.g., $[3,4,6,7,10,21,25]$, the results on the simultaneous identification of the source term $f$ and the initial condition $u(0)$ are very scarce. In $[13,18,23]$ the one-dimensional inverse heat problem (1.4)-(1.5) is studied. In [13] the authors use the boundary element method to recover the space-dependent heat source and the initial data simultaneously. In [23] the authors propose a numerical algorithm based on the method of fundamental solutions. In [18], motivated by the idea of [20] for solving the backward problem of heat equation, a regularization problem is constructed and regularized solutions are obtained.

For the abstract parabolic equation, to our knowledge there is only one result [1] concerning the simultaneous identification of $f$ and $u(0)$. Indeed in [1] G. Bastay applied an iterative alternative method to reconstruct the both functions in the problem (1.2)-(1.3), in the case $A$ has continuous spectrum, however the autor only established theorytical results and didn't give numerical implementation. For this reason, we propose a modified Tikhonov regularization method to recover $f$ and $u(0)$ from additional information given at $t=T_{1}$ and $t=T_{2}$, where the choice of the regularization parameter is based on some a priori knowledge about the magnitude of the errors in the data and we complete our investigation by numerical simulations justifying the feasibility of our approach.

The paper is organized as follows, in section 2 we give some tools which are useful for this study and we show the unstability of the inverse problem. In section 3, we present a modified Tikhonov method and give convergence estimates. The numerical implementation is described in section 4 to illustrate the accuracy and efficiency of the proposed method.

\section{Preliminaries}

Let $\left(\varphi_{n}\right)_{n \geq 1} \subset H$ be an orthonormal eigenbasis corresponding to the eigenvalues $\left(\lambda_{n}\right)_{n \geq 1}$ such that

$$
\begin{gathered}
A \varphi_{n}=\lambda_{n} \varphi_{n}, \quad n \in \mathbb{N}^{*}, \\
0<\lambda_{1} \leq \lambda_{2} \ldots \leq \ldots, \quad \lim _{n \rightarrow \infty} \lambda_{n}=+\infty, \\
\forall b \in H, \quad b=\sum_{n=1}^{\infty} b_{n} \varphi_{n}, \quad b_{n}=\left(b, \varphi_{n}\right) .
\end{gathered}
$$


For $p \in \mathbb{R}$, we introduce the Hilbert spaces $H^{p}$ induced by $A$ as follows

$$
H^{p}=\left\{b \in H: \sum_{n=1}^{\infty}\left(1+\lambda_{n}^{2}\right)^{p}\left|\left(b, \varphi_{n}\right)\right|^{2}<+\infty\right\},
$$

with the norm

$$
\|b\|_{H^{P}}=\left(\sum_{n=1}^{\infty}\left(1+\lambda_{n}^{2}\right)^{p}\left|\left(b, \varphi_{n}\right)\right|^{2}\right)^{\frac{1}{2}}, \quad b \in H^{p} .
$$

We denote by $\left\{S(t)=e^{-t A}\right\}_{t \geq 0}$ the $C_{0}$-semigroup generated by $-A$ on $H$,

$$
S(t) b=e^{-t A} b=\sum_{n=1}^{\infty} e^{-t \lambda_{n}}\left(b, \varphi_{n}\right) \varphi_{n}, \quad \forall b \in H
$$

Theorem 2.1. [17] For the family of operators $\{S(t)\}_{t \geq 0}$, we have the following properties

- $\|S(t)\| \leq 1$, for every $t \geq 0$;

- the function $t \rightarrow S(t), \quad t>0$ is analytic;

- $S(t): H \rightarrow D\left(A^{r}\right)$, for every $t>0$ and $r \geq 0$;

- For every $b \in D\left(A^{r}\right)$ and $r \geq 0, S(t) A^{r} b=A^{r} S(t) b$;

- For every $t>0$ and $r \geq 0$, the operator $A^{r} S(t)$ is bounded.

We end this section by a result concerning the existence and uniqueness of solution of the direct problem.

Theorem 2.2. [8] For given $f \in H$ and $g \in H$ the problem (1.2) has a unique solution $u \in C([0, T), H) \cap C^{1}((0, T), H)$ given by

$$
u=S(t) g+K(t) f=e^{-t A} g+A^{-1}\left(I-e^{-t A}\right) f .
$$

Moreover if $g \in D(A), u \in C^{1}([0, T), H)$.

\subsection{Unstability of the inverse problem}

Now, we wish to solve the inverse problem i.e., find the pair of functions $(f, g)$ in the system (1.2)-(1.3). Making use of the supplementary conditions (1.3), we have

$$
\left\{\begin{array}{l}
u\left(T_{1}\right)=S\left(T_{1}\right) g+K\left(T_{1}\right) f=\psi_{1}, \\
u\left(T_{2}\right)=S\left(T_{2}\right) g+K\left(T_{2}\right) f=\psi_{2} .
\end{array}\right.
$$


Hence, we look for a solution $(f, g)$ to the system

$$
\left\{\begin{array}{l}
S\left(T_{1}\right) g+K\left(T_{1}\right) f=S\left(T_{1}\right) g+A^{-1}\left(I-S\left(T_{1}\right)\right) f=\psi_{1}, \\
S\left(T_{2}\right) g+K\left(T_{2}\right) f=S\left(T_{2}\right) g+A^{-1}\left(I-S\left(T_{2}\right)\right) f=\psi_{2} .
\end{array}\right.
$$

Applying the operator $S\left(T_{2}\right)$ to the first equation in the system (2.1) and the operator $S\left(T_{1}\right)$ to the second one, we have

$$
\begin{aligned}
& S\left(T_{2}\right) S\left(T_{1}\right) g+S\left(T_{2}\right) A^{-1}\left(I-S\left(T_{1}\right)\right) f=S\left(T_{2}\right) \psi_{1}, \\
& S\left(T_{1}\right) S\left(T_{2}\right) g+S\left(T_{1}\right) A^{-1}\left(I-S\left(T_{2}\right)\right) f=S\left(T_{1}\right) \psi_{2} .
\end{aligned}
$$

By subtracting the equation (2.2) from the equation (2.3) and using semigroups properties, we obtain

$$
A^{-1}\left(S\left(T_{1}\right)-S\left(T_{2}\right)\right) f=S\left(T_{1}\right) \psi_{2}-S\left(T_{2}\right) \psi_{1} .
$$

On the other hand, we apply the operator $K\left(T_{2}\right)$ to the first equation in the system (2.1) and $K\left(T_{2}\right)$ to the second one, we have

$$
\begin{aligned}
& K\left(T_{2}\right) S\left(T_{1}\right) g+K\left(T_{2}\right) K\left(T_{1}\right) f=K\left(T_{2}\right) \psi_{1}, \\
& K\left(T_{1}\right) S\left(T_{2}\right) g+K\left(T_{1}\right) K\left(T_{2}\right) f=K\left(T_{1}\right) \psi_{2} .
\end{aligned}
$$

We subtract the equation (2.5) from (2.4), it follows

$$
A^{-1}\left(I-S\left(T_{2}\right)\right) S\left(T_{1}\right) g-A^{-1}\left(I-S\left(T_{1}\right)\right) S\left(T_{2}\right) g=K\left(T_{2}\right) \psi_{1}-K\left(T_{1}\right) \psi_{2},
$$

that is

$$
A^{-1}\left(S\left(T_{1}\right)-S\left(T_{2}\right)\right) g=K\left(T_{2}\right) \psi_{1}-K\left(T_{1}\right) \psi_{2} .
$$

Hence, (2.1) is equivalent to the system

$$
\left\{\begin{array}{l}
B f=\eta_{1}, \\
B g=\eta_{2}
\end{array}\right.
$$

where

$$
\begin{gathered}
B=A^{-1}\left(S\left(T_{1}\right)-S\left(T_{2}\right)\right), \\
\eta_{1}=S\left(T_{1}\right) \psi_{2}-S\left(T_{2}\right) \psi_{1} \quad \text { and } \quad \eta_{2}=K\left(T_{2}\right) \psi_{1}-K\left(T_{1}\right) \psi_{2} .
\end{gathered}
$$

It is easily seen that $B$ is a linear, injective, compact and self-adjoint operator with the singular values $\left(\sigma_{n}=\frac{e^{-T_{1} \lambda_{n}}-e^{-T_{2} \lambda_{n}}}{\lambda_{n}}\right)_{n=1}^{+\infty}$. 
Remark 2.1. As many boundary inverse value problems for partial differential equations which are ill-posed, the study of the problem (1.2)-(1.3) is reduced to the study of the system (2.6), i.e., the study of operator equations of the first kind in $H$, of the form

$$
B b=\eta
$$

From the injectivity of $B$, we obtain

$$
b=B^{-1} \eta=\sum_{n=1}^{\infty} \frac{1}{\sigma_{n}}\left(\eta, \varphi_{n}\right) \varphi_{n} .
$$

Since $\frac{1}{\sigma_{n}} \rightarrow \infty$ as $n \rightarrow \infty$, the inverse problem is ill-posed i.e., the solution does not depend continuously on the given data. Moreover, since in practice the measured data $\psi_{1}$ and $\psi_{2}$ are never known exactly, it is our aim to construct stable approximate solutions of $f$ and $g$ in the system

$$
\left\{\begin{array}{l}
B f=\eta_{1}^{\delta}, \\
B g=\eta_{2}^{\delta}
\end{array}\right.
$$

where $\eta_{1}^{\delta}=S\left(T_{1}\right) \psi_{2}^{\delta}-S\left(T_{2}\right) \psi_{1}^{\delta}, \eta_{2}^{\delta}=K\left(T_{2}\right) \psi_{1}^{\delta}-K\left(T_{1}\right) \psi_{2}^{\delta}, \psi_{1}^{\delta}$ and $\psi_{2}^{\delta}$ are the perturbed data functions satisfying

$$
\left\|\psi_{1}-\psi_{1}^{\delta}\right\|+\left\|\psi_{2}-\psi_{2}^{\delta}\right\| \leq \delta_{1}+\delta_{2}=\delta
$$

here $\delta>0$ denotes a noise level.

Before introducing our results, we require the following assumption, the source term $f$ and the initial condition $u(0)=g$ satisfy the a priori bounds

$$
\begin{aligned}
& \|f\|_{H^{p_{1}}} \leq E_{1}, \quad p_{1}>0 \\
& \|g\|_{H^{p_{2}}} \leq E_{2}, \quad p_{2}>0
\end{aligned}
$$

where $E_{1}, E_{2}>0$ are constants.

\section{A modified Tikhonov regularization method and con- vergence results}

The Tikhonov regularization is a very effective method for solving many illposed problems. However, for this method, it is quite difficult to obtain an explicit error estimate for some complicated problems. In this section, we will propose a modified Tikhonov method for solving the system (2.8). As 
it is known the Tikhonov regulariztion method consists in minimizing the following quantities

$$
\left\{\begin{array}{l}
\left\|B f-\eta_{1}^{\delta}\right\|^{2}+\alpha^{2}\|f\|^{2}, \\
\left\|B g-\eta_{2}^{\delta}\right\|^{2}+\alpha^{2}\|g\|^{2},
\end{array}\right.
$$

with respect to $f$ and $g$ respectively. As it is shown in [15], the unique solution $(f, g)$ of the minimization problems in (3.1) is equal to solve the following normal equations

$$
\left\{\begin{array}{l}
\alpha^{2} f_{\alpha}^{\delta}+B^{*} B f_{\alpha}^{\delta}=B^{*} \eta_{1}^{\delta}, \\
\alpha^{2} g_{\alpha}^{\delta}+B^{*} B g_{\alpha}^{\delta}=B^{*} \eta_{2}^{\delta} .
\end{array}\right.
$$

Since $B$ is a linear self-adjoint operator, we get

$$
\left\{\begin{array}{l}
f_{\alpha}^{\delta}=\left(\alpha^{2} I+B^{2}\right)^{-1} B \eta_{1}^{\delta}, \\
g_{\alpha}^{\delta}=\left(\alpha^{2} I+B^{2}\right)^{-1} B \eta_{2}^{\delta} .
\end{array}\right.
$$

Due to the spectral decomposition for compact self-adjoint operators, we have

$$
\left\{\begin{array}{l}
f_{\alpha}^{\delta}=\sum_{n=1}^{\infty} \frac{\sigma_{n}}{\alpha^{2}+\sigma_{n}^{2}}\left(\eta_{1}^{\delta}, \varphi_{n}\right) \varphi_{n}=\sum_{n=1}^{\infty} \frac{\beta_{n}}{1+\alpha^{2} \beta_{n}^{2}}\left(\eta_{1}^{\delta}, \varphi_{n}\right) \varphi_{n} \\
g_{\alpha}^{\delta}=\sum_{n=1}^{\infty} \frac{\sigma_{n}}{\alpha^{2}+\sigma_{n}^{2}}\left(\eta_{2}^{\delta}, \varphi_{n}\right) \varphi_{n}=\sum_{n=1}^{\infty} \frac{\beta_{n}}{1+\alpha^{2} \beta_{n}^{2}}\left(\eta_{2}^{\delta}, \varphi_{n}\right) \varphi_{n}
\end{array}\right.
$$

where $\beta_{n}=\frac{1}{\sigma_{n}}=\frac{\lambda_{n}}{e^{-T_{1} \lambda_{n}}-e^{-T_{2} \lambda_{n}}}$.

The filter in (3.4) attenuates the coefficients $\left(\eta_{1}^{\delta}, \varphi_{n}\right)$ and $\left(\eta_{2}^{\delta}, \varphi_{n}\right)$ in a manner consistent with the goal of minimizing quantities in (3.1). By this idea, we can use a much better filter $\frac{1}{1+\alpha^{2}\left(\lambda_{n} e^{T_{1} \lambda_{n}}\right)^{2}}$ to replace the filter $\frac{1}{1+\alpha^{2} \beta_{n}^{2}}=$ $\frac{1}{1+\alpha^{2}\left(\frac{\lambda_{n} e^{T_{1} \lambda_{n}}}{1-e^{-\left(T_{2}-T_{1}\right) \lambda_{n}}}\right)^{2}}$ and give other approximations $f_{\alpha}^{\delta}$ and $g_{\alpha}^{\delta}$ of the solutions $f$ and $g$ respectively.

Hence, we define regularization approximate solutions of problem (1.2)-(1.3) which are called the modified Tikhonov regularized solutions as follows

$$
\left\{\begin{array}{l}
f_{\alpha}^{\delta}=\sum_{n=1}^{\infty} \frac{\beta_{n}}{1+\alpha^{2}\left(\lambda_{n} e^{T_{1} \lambda_{n}}\right)^{2}}\left(\eta_{1}^{\delta}, \varphi_{n}\right) \varphi_{n} \\
g_{\alpha}^{\delta}=\sum_{n=1}^{\infty} \frac{\beta_{n}}{1+\alpha^{2}\left(\lambda_{n} e^{T_{1} \lambda_{n}}\right)^{2}}\left(\eta_{2}^{\delta}, \varphi_{n}\right) \varphi_{n}
\end{array}\right.
$$

In the following, we introduce some properties and tools which are useful for our main theorem. 
Lemma 3.1. The norm of the operator $K(t)=A^{-1}\left(I-e^{-t A}\right)$ is given by

$$
\|K(t)\|=\frac{1-e^{-t \lambda_{1}}}{\lambda_{1}}
$$

Proof. Since $\|K(t)\|=\sup _{n \geq 1} \frac{1-e^{-t \lambda_{n}}}{\lambda_{n}}$, we aim to find the spremum of the function $\frac{1-e^{-t \lambda_{n}}}{\lambda_{n}}, n \in \mathbb{N}^{*}$, for this purpose, fixing $t$, letting $\mu=t \lambda$ and defining the function

$$
F_{1}(\mu)=\frac{1-e^{-\mu}}{\mu}, \quad \text { for } \quad \mu \geq \mu_{1}=t \lambda_{1} .
$$

We compute

$$
F_{1}^{\prime}(\mu)=\frac{(\mu+1) e^{-\mu}-1}{\mu^{2}} .
$$

Putting

$$
F_{2}(\mu)=(\mu+1) e^{-\mu}-1
$$

Hence

$$
F_{1}^{\prime}(\mu)=\frac{F_{2}(\mu)}{\mu^{2}} .
$$

To study the monotony of $F_{1}$, it suffice to determine the sign of $F_{2}$. We have

$$
F_{2}^{\prime}(\mu)=-\mu e^{-\mu}<0, \quad \forall \mu \geq \mu_{1}>0,
$$

then, $F_{2}$ is decreasing, moreover $\left.F_{2}(\mu) \subset\right]-1,0\left[\right.$, hence $F_{2}(\mu)<0, \quad \forall \mu \geq \mu_{1}$, which implies that $F_{1}$ is decreasing and

$$
\sup _{\mu \geq \mu_{1}} F_{1}(\mu)=F_{1}\left(\mu_{1}\right)
$$

Therefore,

$$
\sup _{n \geq 1} \frac{1-e^{-t \lambda_{n}}}{\lambda_{n}}=\frac{1-e^{-t \lambda_{1}}}{\lambda_{1}}
$$

Moreover

$$
\sup _{t \in\left[0, T_{2}\right]}\|K(t)\|=\sup _{t \in\left[0, T_{2}\right]} \frac{1-e^{-t \lambda_{1}}}{\lambda_{1}} \leq \frac{1}{\lambda_{1}} .
$$

Lemma 3.2. For $0<\alpha<1$ and $p>0$, the following inequalities hold:

$$
\begin{gathered}
\sup _{n \geq 1}\left(1-\frac{1}{1+\alpha^{2} \lambda_{n}^{2} e^{2 \lambda_{n} T_{1}}}\right)\left(1+\lambda_{n}^{2}\right)^{\frac{-p}{2}} \leq \max \left(1, T_{1}^{p-2}, T_{1}^{p}\right) \max \left(\alpha,\left(\ln \left(\frac{1}{\sqrt{\alpha}}\right)\right)^{-p}\right), \\
\sup _{n \geq 1} \frac{\beta_{n} e^{-\lambda_{n} T_{i}}}{1+\alpha^{2} \lambda_{n}^{2} e^{2 \lambda_{n} T_{1}}} \leq \max \left(1, T_{1}^{-1}\right) \frac{\gamma}{\sqrt{\alpha}}, i=1,2,
\end{gathered}
$$




$$
\sup _{n \geq 1} \frac{\beta_{n}}{\left(1+\alpha^{2} \lambda_{n}^{2} e^{2 \lambda_{n} T_{1}}\right) \lambda_{n}} \leq \max \left(1, \lambda_{1}^{-2}\right) \frac{\gamma}{\alpha},
$$

where $\gamma=\frac{1}{1-e^{-\lambda_{1}\left(T_{2}-T_{1}\right)}}$.

Proof. Let $\lambda_{n 0}=\frac{1}{2 T_{1}} \ln \frac{1}{\alpha}$, for large values of $\lambda_{n}$, that is

- $\lambda_{n} \geq \lambda_{n 0}$, we obtain

$$
\begin{aligned}
G\left(\lambda_{n}\right) & =\left(1-\frac{1}{1+\alpha^{2} \lambda_{n}^{2} e^{2 \lambda_{n} T_{1}}}\right)\left(1+\lambda_{n}^{2}\right)^{-\frac{p}{2}} \\
& \leq\left(1+\lambda_{n}^{2}\right)^{-\frac{p}{2}} \leq \lambda_{n}^{-p} \leq \lambda_{n 0}^{-p}=T_{1}^{p}\left(\ln \left(\frac{1}{\sqrt{\alpha}}\right)\right)^{-p}
\end{aligned}
$$

- For $\lambda_{1} \leq \lambda_{n}<\lambda_{n 0}$, we have

$$
\begin{aligned}
G\left(\lambda_{n}\right) & =\left(\frac{\alpha^{2} \lambda_{n}^{2} e^{2 \lambda_{n} T_{1}}}{1+\alpha^{2} \lambda_{n}^{2} e^{2 \lambda_{n} T_{1}}}\right)\left(1+\lambda_{n}^{2}\right)^{-\frac{p}{2}} \\
& \leq \alpha^{2} \lambda_{n}^{2} e^{2 \lambda_{n} T_{1}}\left(1+\lambda_{n}^{2}\right)^{-\frac{p}{2}}
\end{aligned}
$$

If $0<p \leq 2$, then

$$
\begin{aligned}
G\left(\lambda_{n}\right) & \leq \alpha^{2} e^{2 \lambda_{n} T_{1}} \lambda_{n}^{2-p} \\
& \leq \alpha^{2} e^{2 \lambda_{n 0} T_{1}} \lambda_{n 0}^{2-p}=\alpha\left(\frac{1}{2 T_{1}}\right)^{2-p}\left(\ln \frac{1}{\alpha}\right)^{2-p} .
\end{aligned}
$$

Using the inequality $\alpha\left(\ln \frac{1}{\alpha}\right)^{2} \leq 1$, it follows

$$
G\left(\lambda_{n}\right) \leq \frac{1}{T_{1}^{2-p}}\left(\frac{1}{2} \ln \frac{1}{\alpha}\right)^{-p}=T_{1}^{p-2}\left(\ln \frac{1}{\sqrt{\alpha}}\right)^{-p} .
$$

If $p>2$, we have

$$
G\left(\lambda_{n}\right) \leq \alpha^{2} e^{2 \lambda_{n} T_{1}} \leq \alpha^{2} e^{2 \lambda_{n 0} T_{1}}=\alpha .
$$

From (3.10), (3.11) and (3.12), it follows (3.7).

Let us establish (3.8). From the inequality

$$
\frac{1}{1-e^{-\lambda_{n}\left(T_{2}-T_{1}\right)}} \leq \frac{1}{1-e^{-\lambda_{1}\left(T_{2}-T_{1}\right)}}=\gamma,
$$

for $i=1,2$, we have

$$
\begin{aligned}
\frac{\beta_{n} e^{-\lambda_{n} T_{i}}}{1+\alpha^{2} \lambda_{n}^{2} e^{2 \lambda_{n} T_{1}}} & =\frac{\lambda_{n} e^{-\lambda_{n}\left(T_{i}-T_{1}\right)}}{\left(1-e^{-\lambda_{n}\left(T_{2}-T_{1}\right)}\right)\left(1+\alpha^{2} \lambda_{n}^{2} e^{2 \lambda_{n} T_{1}}\right)} \\
& \leq \frac{\gamma \lambda_{n} e^{-\lambda_{n}\left(T_{i}-T_{1}\right)}}{1+\alpha^{2} \lambda_{n}^{2} e^{2 \lambda_{n} T_{1}}} \\
& \leq \frac{\gamma \lambda_{n}}{1+\alpha^{2} \lambda_{n}^{2} e^{2 \lambda_{n} T_{1}}} .
\end{aligned}
$$


- If $\lambda_{1} \leq \lambda_{n}<\lambda_{n 0}$, from (3.14) it follows that

$$
\frac{\beta_{n} e^{-\lambda_{n} T_{i}}}{1+\alpha^{2} \lambda_{n}^{2} e^{2 \lambda_{n} T_{1}}} \leq \gamma \lambda_{n} \leq \gamma \lambda_{n 0}=\frac{\gamma}{T_{1}} \ln \frac{1}{\sqrt{\alpha}} .
$$

Since for $0<\alpha<1, \ln \left(\frac{1}{\sqrt{\alpha}}\right) \leq \frac{1}{\sqrt{\alpha}}$, we can write

$$
\frac{\beta_{n} e^{-\lambda_{n} T_{i}}}{1+\alpha^{2} \lambda_{n}^{2} e^{2 \lambda_{n} T_{1}}} \leq \frac{\gamma T_{1}^{-1}}{\sqrt{\alpha}} .
$$

- If $\lambda_{n} \geq \lambda_{n 0}$, from (3.14), we have

$$
\begin{aligned}
\frac{\beta_{n} e^{-\lambda_{n} T_{i}}}{1+\alpha^{2} \lambda_{n}^{2} e^{2 \lambda_{n} T_{1}}} & \leq \frac{\gamma \lambda_{n}}{1+\alpha^{2} \lambda_{n}^{2} e^{2 \lambda_{n} T_{1}}} \\
& \leq \frac{\gamma \lambda_{n}}{1+\alpha^{2} \lambda_{n}^{2} e^{2 \lambda_{n} T_{1}}}=\frac{\gamma \lambda_{n}}{1+\alpha \lambda_{n}^{2}}, \quad i=1,2 .
\end{aligned}
$$

In the following, we consider the both cases $T_{1} \geq 1$ and $T_{1}<1$.

- Let $T_{1} \geq 1$, it is clear that $\lambda_{n 1}=\frac{1}{\sqrt{\alpha}}$ is a maximal value point of $G_{1}\left(\lambda_{n}\right)=\frac{\lambda_{n}}{1+\alpha \lambda_{n}^{2}}$, since

$$
\lambda_{n 1}=\frac{1}{\sqrt{\alpha}} \geq \frac{1}{T_{1}} \frac{1}{\sqrt{\alpha}} \geq \lambda_{n 0}=\frac{1}{T_{1}} \ln \left(\frac{1}{\sqrt{\alpha}}\right),
$$

from (3.17) we obtain

$$
\begin{aligned}
\frac{\beta_{n} e^{-\lambda_{n} T_{i}}}{1+\alpha^{2} \lambda_{n}^{2} e^{2 \lambda_{n} T_{1}}} & \leq \gamma G_{1}\left(\lambda_{n}\right) \\
& \leq \gamma G_{1}\left(\lambda_{n 1}\right)=\frac{\gamma \lambda_{n 1}}{1+\alpha \lambda_{n 1}^{2}} \\
& \leq \gamma \lambda_{n 1}=\frac{\gamma}{\sqrt{\alpha}}, \quad i=1,2 .
\end{aligned}
$$

-Let $T_{1}<1$, putting $G_{2}\left(\lambda_{n}\right)=\frac{\lambda_{n}}{1+\alpha T_{1}^{2} \lambda_{n}^{2}}$, from (3.17) we have

$$
\frac{\beta_{n} e^{-\lambda_{n} T_{i}}}{1+\alpha^{2} \lambda_{n}^{2} e^{2 \lambda_{n} T_{1}}} \leq \gamma G_{1}\left(\lambda_{n}\right) \leq \gamma G_{2}\left(\lambda_{n}\right) .
$$

It is clear that $\lambda_{n 2}=\frac{1}{T_{1} \sqrt{\alpha}} \geq \lambda_{n 0}$ is a maximal value point of $G_{2}\left(\lambda_{n}\right)$, hence from (3.19) it follows

$$
\begin{aligned}
\frac{\beta_{n} e^{-\lambda_{n} T_{i}}}{1+\alpha^{2} \lambda_{n}^{2} e^{2 \lambda_{n} T_{1}}} & \leq \gamma G_{2}\left(\lambda_{n 2}\right)=\frac{\gamma \lambda_{n 2}}{1+\alpha T_{1}^{2} \lambda_{n 2}^{2}} \\
& \leq \gamma \lambda_{n 2}=\frac{\gamma}{T_{1} \sqrt{\alpha}} .
\end{aligned}
$$


Combining (3.16), (3.18) and (3.20), it follows (3.8). Let us establish (3.9).

- If $\lambda_{1} \leq \lambda_{n}<\lambda_{n 0}$, we have

$$
\frac{\beta_{n}}{\left(1+\alpha^{2} \lambda_{n}^{2} e^{2 \lambda_{n} T_{1}}\right) \lambda_{n}} \leq \lambda_{n}^{-1} \beta_{n}=\frac{\lambda_{n} e^{\lambda_{n} T_{1}}}{\left(1-e^{\lambda_{n}\left(T_{2}-T_{1}\right)}\right) \lambda_{n}} .
$$

Using the inequalities (3.13) and $\frac{1}{\sqrt{\alpha}}<\frac{1}{\alpha}$, we obtain

$$
\begin{aligned}
\frac{\beta_{n}}{\left(1+\alpha^{2} \lambda_{n}^{2} e^{2 \lambda_{n} T_{1}}\right) \lambda_{n}} & \leq \gamma e^{\lambda_{n} T_{1}} \\
& \leq \gamma e^{\lambda_{n 0} T_{1}}=\frac{\gamma}{\sqrt{\alpha}} \leq \frac{\gamma}{\alpha} .
\end{aligned}
$$

- If $\lambda_{n} \geq \lambda_{n 0}$, we get

$$
\begin{aligned}
\frac{\beta_{n}}{\left(1+\alpha^{2} \lambda_{n}^{2} e^{2 \lambda_{n} T_{1}}\right) \lambda_{n}} & \leq \frac{\gamma e^{\lambda_{n} T_{1}}}{\left(1+\alpha^{2} \lambda_{n}^{2} e^{2 \lambda_{n} T_{1}}\right)} \\
& \leq \frac{\gamma e^{\lambda_{n} T_{1}}}{\left(1+\alpha^{2} \lambda_{1}^{2} e^{2 \lambda_{n} T_{1}}\right)} \\
& \leq \frac{\gamma}{\min \left(1, \lambda_{1}^{2}\right)} \frac{e^{\lambda_{n} T_{1}}}{\left(1+\alpha^{2} e^{2 \lambda_{n} T_{1}}\right)} .
\end{aligned}
$$

It is easy to check that $2 \lambda_{n 0}=\frac{1}{T_{1}} \ln \frac{1}{\alpha}$ is a maximal value point of $G_{3}\left(\lambda_{n}\right)=\frac{e^{\lambda_{n} T_{1}}}{\left(1+\alpha^{2} e^{2 \lambda_{n} T_{1}}\right)}$, so

$$
\begin{aligned}
\frac{\beta_{n}}{\left(1+\alpha^{2} \lambda_{n}^{2} e^{2 \lambda_{n} T_{1}}\right) \lambda_{n}} & \leq \gamma \max \left(1, \lambda_{1}^{-2}\right) G_{3}\left(2 \lambda_{n 0}\right) \\
& \leq \gamma \max \left(1, \lambda_{1}^{-2}\right) e^{2 \lambda_{n 0} T_{1}} \\
& \leq \max \left(1, \lambda_{1}^{-2}\right) \frac{\gamma}{\alpha} .
\end{aligned}
$$

From (3.21) and (3.23), the inequality (3.9) is obtained.

Theorem 3.3. Let $f_{\alpha}^{\delta}$ and $g_{\alpha}^{\delta}$ be the modified Tikhonov approximations of the solutions $f$ and $g$ of problem (1.2)-(1.3) such that (2.10) and (2.11) hold. Let $\psi_{1}^{\delta}$ and $\psi_{2}^{\delta}$ be the measured data at $T_{1}$ and $T_{2}$ respectively, $\left(0<T_{1}<T_{2}\right)$ satisfying (2.9). If the regularization parameter is chosen as $\alpha=\left(\frac{\delta}{E_{1}}\right)^{2 /\left(p_{1}+2\right)}$ and $\alpha=\left(\frac{\delta}{E_{2}}\right)^{2 /\left(p_{2}+2\right)}$ respectively then, the following error estimates hold respectively

$$
\begin{aligned}
\left\|f-f_{\alpha}^{\delta}\right\| & \leq \max \left(1, T_{1}^{p_{1}-2}, T_{1}^{p_{1}}\right) \max \left(\left(\frac{\delta}{E_{1}}\right)^{\frac{2}{p_{1}+2}}, \frac{1}{\left(\ln \left(\frac{E_{1}}{\delta}\right)^{\frac{1}{\left(p_{1}+2\right)}}\right)^{p_{1}}}\right) E_{1} \\
& +\gamma \max \left(1, T_{1}^{-1}\right)\left(\frac{\delta}{E_{1}}\right)^{\frac{p_{1}+1}{\left(p_{1}+2\right)}} E_{1}^{\frac{p_{1}}{\left(p_{1}+2\right)}}
\end{aligned}
$$




$$
\begin{aligned}
\left\|g-g_{\alpha}^{\delta}\right\| & \leq \max \left(1, T_{1}^{p_{2}-2}, T_{1}^{p_{2}}\right) \max \left(\left(\frac{\delta}{E_{2}}\right)^{\frac{2}{p_{2}+2}}, \frac{1}{\left(\ln \left(\frac{E_{2}}{\delta}\right)^{\frac{1}{\left(p_{2}+2\right)}}\right) p_{2}}\right) E_{2} \\
& +\gamma \max \left(1, \lambda_{1}^{-2}\right)\left(\frac{\delta}{E_{2}}\right)^{\frac{p_{2}}{p_{2}+2}} E_{2}^{\frac{2+p_{2}}{\left(p_{2}+2\right)}}
\end{aligned}
$$

Proof. Let us prove (3.24). Apply the triangle inequality, we have

$$
\left\|f-f_{\alpha}^{\delta}\right\| \leq\left\|f-f_{\alpha}\right\|+\left\|f_{\alpha}-f_{\alpha}^{\delta}\right\| .
$$

We compute

$$
\begin{aligned}
\left\|f-f_{\alpha}\right\| & =\left\|\sum_{n=1}^{\infty} \beta_{n}\left(\eta_{1}, \varphi_{n}\right) \varphi_{n}-\sum_{n=1}^{\infty} \frac{\beta_{n}}{1+\alpha^{2} \lambda_{n}^{2} e^{2 \lambda_{n} T_{1}}}\left(\eta_{1}, \varphi_{n}\right) \varphi_{n}\right\| \\
& =\left\|\sum_{n=1}^{\infty}\left(1-\frac{1}{1+\alpha^{2} \lambda_{n}^{2} e^{2 \lambda_{n} T_{1}}}\right)\left(1+\lambda_{n}^{2}\right)^{-p_{1} / 2}\left(1+\lambda_{n}^{2}\right)^{p_{1} / 2}\left(f, \varphi_{n}\right) \varphi_{n}\right\| \\
& \leq \sup _{n \geq 1}\left(\left(1-\frac{1}{1+\alpha^{2} \lambda_{n}^{2} e^{2 \lambda_{n} T_{1}}}\right)\left(1+\lambda_{n}\right)^{\frac{-p_{1}}{2}}\right)\left\|\sum_{n=1}^{\infty}\left(1+\lambda_{n}^{2}\right)^{p_{1} / 2}\left(f, \varphi_{n}\right) \varphi_{n}\right\| .
\end{aligned}
$$

From inequality (3.7) and (2.10) it follows that

$$
\left\|f-f_{\alpha}\right\| \leq \max \left(1, T_{1}^{p_{1}-2}, T_{1}^{p_{1}}\right) \max \left(\alpha,\left(\ln \left(\frac{1}{\sqrt{\alpha}}\right)\right)^{-p_{1}}\right) E_{1} .
$$

On the other hand

$$
\begin{aligned}
\left\|f_{\alpha}-f_{\alpha}^{\delta}\right\| & =\left\|\sum_{n=1}^{\infty} \frac{\beta_{n}}{1+\alpha^{2} \lambda_{n}^{2} e^{2 \lambda_{n} T_{1}}}\left(\eta_{1}, \varphi_{n}\right) \varphi_{n}-\sum_{n=1}^{\infty} \frac{\beta_{n}}{1+\alpha^{2} \lambda_{n}^{2} e^{2 \lambda_{n} T_{1}}}\left(\eta_{1}^{\delta}, \varphi_{n}\right) \varphi_{n}\right\| \\
& =\left\|\sum_{n=1}^{\infty} \frac{\beta_{n}}{1+\alpha^{2} \lambda_{n}^{2} e^{2 \lambda_{n} T_{1}}}\left(\eta_{1}-\eta_{1}^{\delta}, \varphi_{n}\right) \varphi_{n}\right\| \\
& =\| \sum_{n=1}^{\infty} \frac{\beta_{n}}{1+\alpha^{2} \lambda_{n}^{2} e^{2 \lambda_{n} T_{1}}} e^{-T_{1} \lambda_{n}}\left(\psi_{2}-\psi_{2}^{\delta}, \varphi_{n}\right) \varphi_{n} \\
& +\sum_{n=1}^{\infty} \frac{\beta_{n}}{1+\alpha^{2} \lambda_{n}^{2} e^{2 \lambda_{n} T_{1}}} e^{-T_{2} \lambda_{n}}\left(\psi_{1}^{\delta}-\psi_{1}, \varphi_{n}\right) \varphi_{n} \| \\
& \leq \sup _{n \geq 1}\left(\frac{\beta_{n} e^{-T_{1} \lambda_{n}}}{1+\alpha^{2} \lambda_{n}^{2} e^{2 \lambda_{n} T_{1}}}\right)\left\|\sum_{n=1}^{\infty}\left(\psi_{2}-\psi_{2}^{\delta}, \varphi_{n}\right) \varphi_{n}\right\| \\
& +\sup _{n \geq 1}\left(\frac{\beta_{n} e^{-T_{2} \lambda_{n}}}{1+\alpha^{2} \lambda_{n}^{2} e^{2 \lambda_{n} T_{1}}}\right)\left\|\sum_{n=1}^{\infty}\left(\psi_{1}-\psi_{1}^{\delta}, \varphi_{n}\right) \varphi_{n}\right\| .
\end{aligned}
$$


Using the estimates (3.8) and (2.9), we obtain

$$
\left\|f_{\alpha}-f_{\alpha}^{\delta}\right\| \leq \max \left(1, T_{1}^{-1}\right) \frac{\gamma}{\sqrt{\alpha}}\left(\delta_{1}+\delta_{2}\right)=\gamma \max \left(1, T_{1}^{-1}\right) \frac{\delta}{\sqrt{\alpha}} .
$$

Combining (3.26) with (3.27) and (3.28), we obtain

$$
\begin{aligned}
\left\|f-f_{\alpha}^{\delta}\right\| & \leq \max \left(1, T_{1}^{p_{1}-2}, T_{1}^{p_{1}}\right) \max \left(\alpha,\left(\ln \left(\frac{1}{\sqrt{\alpha}}\right)\right)^{-p_{1}}\right) E_{1}+ \\
& +\gamma \max \left(1, T_{1}^{-1}\right) \frac{\delta}{\sqrt{\alpha}} .
\end{aligned}
$$

If we select $\alpha=\left(\frac{\delta}{E_{1}}\right)^{2 /\left(p_{1}+2\right)}$, then one has

$$
\begin{aligned}
\left\|f-f_{\alpha}^{\delta}\right\| & \leq \max \left(1, T_{1}^{p_{1}-2}, T_{1}^{p_{1}}\right) \max \left(\left(\frac{\delta}{E_{1}}\right)^{\frac{2}{p_{1}+2}},\left(\ln \left(\frac{E_{1}}{\delta}\right)^{\frac{1}{\left(p_{1}+2\right)}}\right)^{-p_{1}}\right) E_{1} \\
& +\gamma \max \left(1, T_{1}^{-1}\right)\left(\frac{\delta}{E_{1}}\right)^{\frac{-1}{\left(p_{1}+2\right)}} \delta \\
& \leq \max \left(1, T_{1}^{p_{1}-2}, T_{1}^{p_{1}}\right) \max \left(\left(\frac{\delta}{E_{1}}\right)^{\frac{2}{p_{1}+2}},\left(\ln \left(\frac{E_{1}}{\delta}\right)^{\frac{1}{\left(p_{1}+2\right)}}\right)^{-p_{1}}\right) E_{1} \\
& +\gamma \max \left(1, T_{1}^{-1}\right)\left(\frac{\delta}{E_{1}}\right)^{\frac{p_{1}+1}{\left(p_{1}+2\right)}} E_{1}^{\frac{p_{1}}{\left(p_{1}+2\right)}} .
\end{aligned}
$$

Now, we prove (3.25), we have

$$
\left\|g-g_{\alpha}^{\delta}\right\| \leq\left\|g-g_{\alpha}\right\|+\left\|g_{\alpha}-g_{\alpha}^{\delta}\right\| .
$$

By the same calculate used to obtain (3.27), it follows

$$
\left\|g-g_{\alpha}\right\| \leq \max \left(1, T_{1}^{p_{2}-2}, T_{1}^{p_{2}}\right) \max \left(\alpha,\left(\ln \left(\frac{1}{\sqrt{\alpha}}\right)\right)^{-p_{2}}\right) E_{2} .
$$


On the other hand

$$
\begin{aligned}
& \left\|g_{\alpha}-g_{\alpha}^{\delta}\right\|=\left\|\sum_{n=1}^{\infty} \frac{\beta_{n}}{1+\alpha^{2} \lambda_{n}^{2} e^{2 \lambda_{n} T_{1}}}\left(\eta_{2}, \varphi_{n}\right) \varphi_{n}-\sum_{n=1}^{\infty} \frac{\beta_{n}}{1+\alpha^{2} \lambda_{n}^{2} e^{2 \lambda_{n} T_{1}}}\left(\eta_{2}^{\delta}, \varphi_{n}\right) \varphi_{n}\right\| \\
& =\left\|\sum_{n=1}^{\infty} \frac{\beta_{n}}{1+\alpha^{2} \lambda_{n}^{2} e^{2 \lambda_{n} T_{1}}}\left(\eta_{2}-\eta_{2}^{\delta}, \varphi_{n}\right) \varphi_{n}\right\| \\
& =\| \sum_{n=1}^{\infty} \frac{\beta_{n}}{1+\alpha^{2} \lambda_{n}^{2} e^{2 \lambda_{n} T_{1}}} \frac{\left(1-e^{-T_{2} \lambda_{n}}\right)}{\lambda_{n}}\left(\psi_{1}-\psi_{1}^{\delta}, \varphi_{n}\right) \varphi_{n} \\
& +\sum_{n=1}^{\infty} \frac{\beta_{n}}{1+\alpha^{2} \lambda_{n}^{2} e^{2 \lambda_{n} T_{1}}} \frac{\left(1-e^{-T_{1} \lambda_{n}}\right)}{\lambda_{n}}\left(\psi_{2}^{\delta}-\psi_{2}, \varphi_{n}\right) \varphi_{n} \| \\
& \leq \sup _{n \geq 1}\left(\frac{\beta_{n}}{\left(1+\alpha^{2} \lambda_{n}^{2} e^{2 \lambda_{n} T_{1}}\right) \lambda_{n}}\right)\left\|\sum_{n=1}^{\infty}\left(\psi_{1}-\psi_{1}^{\delta}, \varphi_{n}\right) \varphi_{n}\right\| \\
& +\sup _{n \geq 1}\left(\frac{\beta_{n}}{\left(1+\alpha^{2} \lambda_{n}^{2} e^{2 \lambda_{n} T_{1}}\right) \lambda_{n}}\right)\left\|\sum_{n=1}^{\infty}\left(\psi_{2}-\psi_{2}^{\delta}, \varphi_{n}\right) \varphi_{n}\right\| .
\end{aligned}
$$

Using the estimates (3.9) and (2.9), we obtain

$$
\left\|g_{\alpha}-g_{\alpha}^{\delta}\right\| \leq \max \left(1, \lambda_{1}^{-2}\right) \frac{\gamma}{\alpha}\left(\delta_{1}+\delta_{2}\right)=\gamma \max \left(1, \lambda_{1}^{-2}\right) \frac{\delta}{\alpha}
$$

Combining (3.30) with (3.31) and (3.32), we obtain

$$
\begin{aligned}
\left\|g-g_{\alpha}^{\delta}\right\| & \leq \max \left(1, T_{1}^{p_{2}-2}, T_{1}^{p_{2}}\right) \max \left(\alpha,\left(\ln \left(\frac{1}{\sqrt{\alpha}}\right)\right)^{-p_{2}}\right) E_{2} \\
& +\gamma \max \left(1, \lambda_{1}^{-2}\right) \frac{\delta}{\alpha} .
\end{aligned}
$$

If we select $\alpha=\left(\frac{\delta}{E_{2}}\right)^{2 /\left(p_{2}+2\right)}$, then one has

$$
\begin{aligned}
\left\|g-g_{\alpha}^{\delta}\right\| & \leq \max \left(1, T_{1}^{p_{2}-2}, T_{1}^{p_{2}}\right) \max \left(\left(\frac{\delta}{E_{2}}\right)^{\frac{2}{p_{2}+2}},\left(\ln \left(\frac{E_{2}}{\delta}\right)^{\frac{1}{\left(p_{2}+2\right)}}\right)^{-p_{2}}\right) E_{2} \\
& +\gamma \max \left(1, \lambda_{1}^{-2}\right)\left(\frac{\delta}{E_{2}}\right)^{\frac{-2}{\left(p_{2}+2\right)}} \delta \\
& \leq \max \left(1, T_{1}^{p_{2}-2}, T_{1}^{p_{2}}\right) \max \left(\left(\frac{\delta}{E_{2}}\right)^{\frac{2}{p_{2}+2}}, \frac{1}{\left(\ln \left(\frac{E_{2}}{\delta}\right)^{\frac{1}{\left(p_{2}+2\right)}}\right)^{p_{2}}}\right) E_{2} \\
& +\gamma \max \left(1, \lambda_{1}^{-2}\right)\left(\frac{\delta}{E_{2}}\right)^{\frac{p_{2}}{p_{2}+2}} E_{2}^{\frac{2+p_{2}}{\left(p_{2}+2\right)}} .
\end{aligned}
$$


Remark 3.1. • In practice $\|f\|_{p}$ is usually not known, so we do not obtain an exact priori bound $E$. However, if we select $\alpha=C \delta^{\frac{2}{p+2}}$, where $\mathrm{C}$ is a positive constant, we can also obtain the convergence results. Indeed, if we choose $\alpha=C(\delta)^{2 /\left(p_{1}+2\right)}$ and $\alpha=C(\delta)^{2 /\left(p_{2}+2\right)}$ respectively, we obtain from $(3.29)$ and (3.33) respectively the following estimates

$$
\begin{aligned}
\left\|f-f_{\alpha}^{\delta}\right\| & \leq \max \left(1, T_{1}^{p_{1}-2}, T_{1}^{p_{1}}\right) \max \left(C \delta^{\frac{2}{p_{1}+2}},\left(\ln \left(\frac{1}{\sqrt{C} \delta^{\frac{1}{p_{1}+2}}}\right)\right)^{-p_{1}}\right) E_{1} \\
& +\gamma \max \left(1, T_{1}^{-1}\right) C^{-1 / 2} \delta^{\frac{p_{1}+1}{p_{1}+2}} \rightarrow 0 \quad \text { as } \quad \delta \rightarrow 0
\end{aligned}
$$

and

$$
\begin{aligned}
\left\|g-g_{\alpha}^{\delta}\right\| & \leq \max \left(1, T_{1}^{p_{2}-2}, T_{1}^{p_{2}}\right) \max \left(C \delta^{\frac{2}{p_{2}+2}},\left(\ln \left(\frac{1}{\sqrt{C} \delta^{\frac{1}{p_{2}+2}}}\right)\right)^{-p_{2}}\right) E_{2} \\
& +\gamma \max \left(1, \lambda^{-2}\right) C^{-1} \delta^{\frac{p_{2}}{p_{2}+2}} \rightarrow 0 \quad \text { as } \quad \delta \rightarrow 0 .
\end{aligned}
$$

Hence, $f_{\alpha}^{\delta}$ and $g_{\alpha}^{\delta}$ can be viewed as the approximations of the exact solutions $f$ and $g$ respectively.

- From the convergence estimates (3.24) and (3.25) we can see that the logarithmic term with respect to $\delta$ is the dominating term. Asymptotically this yields a convergence rate of order $\left(\ln \left(\frac{E}{\delta}\right)^{\frac{1}{(p+2)}}\right)^{-p}$, the others terms are asymptotically negligible compared to this term.

\section{Numerical implementation}

In this section, we will numerically implement two examples to illustrate the effectiveness of the proposed method. Consider the problem of finding the functions $f(x), g(x)$ and $u(x, t)$ in the system

$$
\left\{\begin{array}{lr}
u_{t}(x, t)-u_{x x}(x, t)=f(x), & 0<x<\pi, 0<t \leq 1 \\
u(x, 0)=g(x), & 0 \leq x \leq \pi \\
u(0, t)=u(\pi, t)=0, & 0<t<1 \\
u\left(x, \frac{1}{2}\right)=\psi_{1}(x), u(x, 1)=\psi_{2}(x), & 0 \leq x \leq \pi
\end{array}\right.
$$

Denote

$$
\begin{gathered}
A=-\frac{\partial^{2}}{\partial x^{2}}, \quad \text { with } \quad \mathcal{D}(A)=H_{0}^{1}(0, \pi) \cap H^{2}(0, \pi) \subset H=L^{2}(0, \pi) . \\
\lambda_{n}=n^{2}, \varphi_{n}=\sqrt{\frac{2}{\pi}} \sin (n x), n \in \mathbb{N}^{*}
\end{gathered}
$$


are eigenvalues and orthonormal eigenfunctions, which form a basis for $H$. By separating varaiables, we obtain the solution of the direct problem corresponding to problem (4.1) as follows

$$
u(x, t)=S(t) g(x)+K(t) f(x)=\sum_{n=1}^{\infty}\left(e^{-n^{2} t}\left(g, \varphi_{n}\right)+\frac{\left(1-e^{-n^{2} t}\right)}{n^{2}}\left(f, \varphi_{n}\right)\right) \varphi_{n},
$$

where for $b \in H, b_{n}=\left(b, \varphi_{n}\right)=\sqrt{\frac{2}{\pi}} \int_{0}^{1} b(s) \sin (n s) d s, n=1,2 \ldots$

The modified Tikhonov regularized solutions are given by

$$
\begin{aligned}
& f_{\alpha}^{\delta}(x)=\sum_{n=1}^{\infty} \frac{\beta_{n}}{1+\alpha^{2} n^{4} e^{n^{2}}}\left(\eta_{1}^{\delta}, \varphi_{n}\right) \varphi_{n} \\
& g_{\alpha}^{\delta}(x)=\sum_{n=1}^{\infty} \frac{\beta_{n}}{1+\alpha^{2} n^{4} e^{n^{2}}}\left(\eta_{2}^{\delta}, \varphi_{n}\right) \varphi_{n}
\end{aligned}
$$

where $\beta_{n}=\frac{n^{2}}{e^{-\frac{n^{2}}{2}}-e^{-n^{2}}}$,

$$
\eta_{1}^{\delta}(x)=\sum_{n=1}^{\infty}\left(e^{-\frac{1}{2} n^{2}} \psi_{2}^{\delta}-e^{-n^{2}} \psi_{1}^{\delta}, \varphi_{n}\right) \varphi_{n}
$$

and

$$
\eta_{2}^{\delta}(x)=\sum_{n=1}^{\infty}\left(\frac{\left(1-e^{-n^{2}}\right)}{n^{2}} \psi_{1}^{\delta}-\frac{\left(1-e^{-\frac{1}{2} n^{2}}\right)}{n^{2}} \psi_{2}^{\delta}, \varphi_{n}\right) \varphi_{n}
$$

Hence, we have

$$
\begin{aligned}
& f_{\alpha}^{\delta}(x)=\int_{0}^{1} \sum_{n=1}^{\infty} \frac{\beta_{n}}{1+\alpha^{2} n^{4} e^{n^{2}}}\left(\theta_{1 n} \psi_{2}^{\delta}(s)-\theta_{2 n} \psi_{1}^{\delta}(s)\right) \sin (n s) \sin (n x) d s, \\
& g_{k}^{\alpha}(x)=\int_{0}^{1} \sum_{n=1}^{\infty} \frac{\beta_{n}}{1+\alpha^{2} n^{4} e^{n^{2}}}\left(\gamma_{1 n} \psi_{1}^{\delta}(s)-\gamma_{2 n} \psi_{2}^{\delta}(s)\right) \sin (n s) \sin (n x) d s,
\end{aligned}
$$

with $\theta_{1 n}=e^{-\frac{1}{2} n^{2}}, \theta_{2 n}=e^{-n^{2}}, \gamma_{1 n}=\frac{\left(1-e^{-n^{2}}\right)}{n^{2}}$, and $\gamma_{2 n}=\frac{\left(1-e^{-\frac{1}{2} n^{2}}\right)}{n^{2}}$.

We use the trapezoidal rule to approach the integral and do an approximate truncation for the series by choosing the sum of the front $M$ the sum of the front $M$ terms. After considering an equidistant grid $0=x_{1}<x_{2}<\ldots$. $<$ $x_{M+1}=\pi, \quad\left(x_{i}=\frac{(i-1) \pi}{M}, i=1, \ldots, M+1\right)$, we get the discrete approximations

$$
f_{\alpha}^{\delta}=\left(f_{\alpha}^{\delta}\left(x_{1}\right), f_{\alpha}^{\delta}\left(x_{2}\right), \ldots, f_{\alpha}^{\delta}\left(x_{M}\right)\right)
$$


and

$$
g_{\alpha}^{\delta}=\left(g_{\alpha}^{\delta}\left(x_{1}\right), g_{\alpha}^{\delta}\left(x_{2}\right), \ldots, g_{\alpha}^{\delta}\left(x_{M}\right)\right)
$$

of (4.2) and (4.3) respectively, given by the followig matrix forms

$$
\begin{aligned}
& f_{\alpha}^{\delta}=\mathbb{A}^{\alpha} \Psi_{2}^{\delta}-\mathbb{B}^{\alpha} \Psi_{1}^{\delta}, \\
& g_{\alpha}^{\delta}=\mathbb{C}^{\alpha} \Psi_{2}^{\delta}-\mathbb{D}^{\alpha} \Psi_{1}^{\delta},
\end{aligned}
$$

where

$$
\begin{aligned}
A_{i j}^{\alpha} & =\frac{2}{\pi} \sum_{n=1}^{N} \frac{\beta_{n} \theta_{1 n}}{1+\alpha^{2}\left(n^{4} e^{n^{2}}\right.} \sin \left(n x_{i}\right) \sin \left(n x_{j}\right) l, \\
B_{i j}^{\alpha} & =\frac{2}{\pi} \sum_{n=1}^{N} \frac{\beta_{n} \theta_{2 n}}{1+\alpha^{2} n^{4} e^{n^{2}}} \sin \left(n x_{i}\right) \sin \left(n x_{j}\right) l, \\
C_{i j}^{\alpha} & =\frac{2}{\pi} \sum_{n=1}^{N} \frac{\beta_{n} \gamma_{1 n}}{1+\alpha^{2} n^{4} e^{n^{2}}} \sin \left(n x_{i}\right) \sin \left(n x_{j}\right) l, \\
D_{i j}^{\alpha} & =\frac{2}{\pi} \sum_{n=1}^{N} \frac{\beta_{n} \gamma_{2 n}}{1+\alpha^{2} n^{4} e^{n^{2}}} \sin \left(n x_{i}\right) \sin \left(n x_{j}\right) l,
\end{aligned}
$$

$l=\frac{\pi}{M}$ and $\Psi_{i}^{\delta} \in \mathbb{R}^{M+1}, i=1,2$ are the vectors obtained by adding a random distributed perturbation to the corresponding data

$$
\Psi_{i}=\left(\psi_{i}\left(x_{1}\right), \psi_{i}\left(x_{2}\right), \ldots, \psi_{i}\left(x_{M}\right)\right)
$$

i.e.,

$$
\Psi_{i}^{\delta}=\Psi_{i}+\varepsilon \operatorname{randn}\left(\operatorname{size}\left(\Psi_{i}\right)\right), i=1,2,
$$

$\varepsilon$ indicates the noise level of the measurements data and the function $\operatorname{randn}(\cdot)$ generates arrays of random numbers whose elements are normally distributed with mean 0 , variance $\sigma^{2}=1$ and standard deviation $\sigma=1$. $\operatorname{randn}(\operatorname{size}(\mathrm{g}))$ returns an array of random entries that is of the same size as $\psi$. The noise level $\delta$ can be measured in the sense of root mean square error (RMSE) according to

$$
\delta=\left\|\psi^{\delta}-\psi\right\|_{l^{2}}=\left(\frac{1}{N+1} \sum_{i=0}^{N}\left(\psi\left(x_{i}\right)-\psi^{\delta}\left(x_{i}\right)\right)^{2}\right)^{1 / 2} .
$$

In some cases the direct problem with the heat source $f(x)$ and the initial condition $g(x)$ does not have an anlytical solution, in this case we propose to discretise numerically the problem using a finite difference method. Considering a uniform time-grid of $\Delta t=\frac{1}{m}, t_{j}=j \Delta t, j=0, \ldots, m$ and a uniform 
space-grid of $h=\frac{1}{n}, x_{i}=i h, i=0, \ldots, n$ and using the following CrankNikolson scheme

$$
\left\{\begin{array}{l}
\frac{u_{i}^{j+1}-u_{i}^{j}}{\Delta t}=\frac{1}{2}\left(\frac{1}{h^{2}}\left(u_{i+1}^{j+1}+u_{i-1}^{j+1}-2 u_{i}^{j+1}\right)+f_{i}\right)+\frac{1}{2}\left(\frac{1}{h^{2}}\left(u_{i+1}^{j}+u_{i-1}^{j}-2 u_{i}^{j}\right)+f_{i}\right), \\
u_{0}^{j}=u_{n}^{j}=0, \quad 1 \leq j \leq m, \\
u_{i}^{0}=g_{i}, \quad 0 \leq i \leq n,
\end{array}\right.
$$

here $u_{i}^{j}$ is the approximate value of $u\left(x_{i}, t_{j}\right)$ at the mesh points $\left(x_{i}, t_{j}\right), f_{i}=$ $f\left(x_{i}\right)$ and $g_{i}=g\left(x_{i}\right)$. Rewriting the resulting system into a matrix form, we obtain the following $(n-1) \times(n-1)$ linear system of equations

$$
\left\{\begin{array}{l}
A U^{j+1}=B U^{j}+C, 0 \leq j \leq m-1, \\
U^{0}=\left(g_{1}, g_{2}, \ldots, g_{n-1}\right)^{\top}
\end{array}\right.
$$

where

$$
\begin{aligned}
& A=\left(\begin{array}{ccccc}
1+\mu & -\frac{\mu}{2} & & & \\
-\frac{\mu}{2} & 1+\mu & -\frac{\mu}{2} & & \\
& \ddots & \ddots & \ddots & \\
& & -\frac{\mu}{2} & 1+\mu & -\frac{\mu}{2} \\
& & & -\frac{\mu}{2} & 1+\mu
\end{array}\right) \\
& B=\left(\begin{array}{ccccc}
1-\mu & \frac{\mu}{2} & & & \\
\frac{\mu}{2} & 1-\mu & \frac{\mu}{2} & & \\
& \ddots & \ddots & \ddots & \\
& & \frac{\mu}{2} & 1-\mu & \frac{\mu}{2} \\
& & & \frac{\mu}{2} & 1-\mu
\end{array}\right)
\end{aligned}
$$

$\mu=\frac{\Delta t}{h^{2}}, U^{j}=\left(u_{1}^{j}, u_{1}^{j}, \ldots, u_{n-1}^{j}\right)^{\top}$ and $C=\left(f_{1}, f_{2}, \ldots, f_{n-2}, f_{n-1}\right)^{\top}$.

In the following we give two examples, the first example haS the exact expression of the solutions $(u(x, t), f(x), g(x))$, in the second example, the solution does not possess an explicit analytical expression.

Example 1 It is easy to see that if $f(x)=2 \sin x$ and $g(x)=\sin x$, then $u(x, t)=\left(2-e^{-t}\right) \sin x$ is the exact solution of the problem (4.1). Consequently, $\psi_{1}(x)=\left(2-e^{-\frac{1}{2}}\right) \sin x$ and $\psi_{2}(x)=\left(2-e^{-1}\right) \sin x$. 
A MODIFIED TIKHONOV REGULARIZATION METHOD FOR A CLASS OF INVERSE PARABOLIC PROBLEMS

\begin{tabular}{|c|c|c|c|c|c|c|c|c|}
\hline$\alpha$ & $0.05 \alpha_{0}$ & $0.1 \alpha_{0}$ & $0.2 \alpha_{0}$ & $0.3 \alpha_{0}$ & $0.4 \alpha_{0}$ & $0.5 \alpha_{0}$ & $0.6 \alpha_{0}$ & $\alpha_{0}$ \\
\hline $\operatorname{Rer}(f)$ & 0.0035 & 0.0017 & 0.0011 & 0.0047 & 0.0033 & 0.0043 & 0.0097 & 0.0172 \\
\hline $\operatorname{Rer}(g)$ & 0.0191 & 0.0041 & 0.0028 & 0.0072 & 0.0160 & 0.0014 & 0.0080 & 0.0139 \\
\hline
\end{tabular}

Table 1. Relative errors $\operatorname{Rer}(f)$ and $\operatorname{Rer}(g)$ with $p=1, M=100, N=6$, $\epsilon=0.01, \delta=0.0216, \alpha_{0}=\delta^{\frac{2}{p+2}}=0.0776$ for example 1 .

\begin{tabular}{|c|c|c|c|c|c|c|c|}
\hline$N$ & 4 & 5 & 6 & 7 & 8 & 9 & 10 \\
\hline $\operatorname{Rer}(f)$ & 0.0040 & 0.0018 & 0.0011 & 0.0052 & 0.0054 & 0.0033 & 0.0045 \\
\hline $\operatorname{Rer}(g)$ & 0.0117 & 0.0037 & 0.0028 & 0.0082 & 0.0143 & 0.0023 & 0.0135 \\
\hline
\end{tabular}

Table 2. Relative errors $\operatorname{Rer}(f)$ and $\operatorname{Rer}(g)$ with $p=1, M=100, \epsilon=0.01$ and $\alpha=\alpha_{0} \cdot 0.2 \simeq 0.0155$ for example 1 .

\begin{tabular}{|c|c|c|c|c|c|l|c|}
\hline$M$ & 10 & 50 & 100 & 150 & 200 & 250 & 300 \\
\hline $\operatorname{Rer}(f)$ & 0.0130 & 0.0051 & 0.0016 & 0.0017 & 0.0023 & 0.022 & 0.0018 \\
\hline $\operatorname{Rer}(g)$ & 0.0320 & 0.0190 & 0.0039 & 0.0072 & 0.0227 & 0.0036 & 0.0075 \\
\hline
\end{tabular}

Table 3. Relative errors $\operatorname{Rer}(f)$ and $\operatorname{Rer}(g)$ with $p=1, N=6, \epsilon=0.01$ for example 1.

\begin{tabular}{|c|c|c|l|c|}
\hline$\epsilon$ & 0.1 & 0.01 & 0.001 & 0.0001 \\
\hline$\alpha$ & 0.3600 & 0.0727 & 0.0149 & 0.0034 \\
\hline $\operatorname{Rer}(f)$ & 0.0463 & 0.0017 & $1.6034 e-004$ & $7.1518 e-005$ \\
\hline $\operatorname{Rer}(g)$ & 0.0503 & 0.0037 & 0.0032 & 0.0015 \\
\hline
\end{tabular}

Table 4. Relative errors $\operatorname{Rer}(f)$ and $\operatorname{Rer}(g)$ with $p=1, M=100, N=6$, for example 1.

Table 1 shows that the parameter $\alpha$ has the regularization effect. So, we can choose $\alpha=0.2 \alpha_{0},\left(\alpha_{0}=\delta^{2 / 3}\right)$. Thus, in practice, it is relatively easy to find an appropriate value of $\alpha$. Tables 2 and 3 and show the influence of the choice of $N$ and $M$ respectively on the relative error. Table 4 and Figure 1 (resp. Figure 2) show the comparisons between the function $f(x)$ (resp. $g(x)$ ) and its computed approximations with different noise level. It can be seen that as the amount of noise decreases the regularized solutions approximate better the exact functions and even with the noise level $\epsilon=0.1$ the approximate solutions are still good agreement with the corresponding exact solutions. 


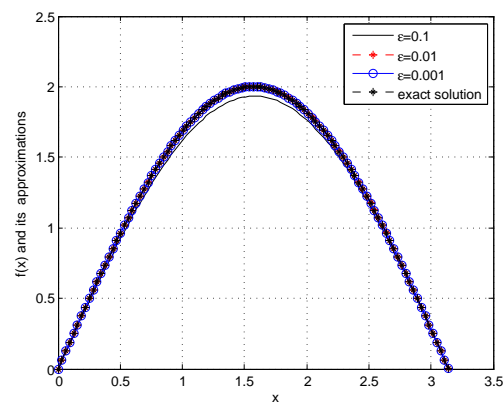

Figure 1: The comparison between the exact solution $f$ and its computed approximations $f_{\delta}^{\epsilon}$ with $M=100, N=6$ and $p=1$ for example1.

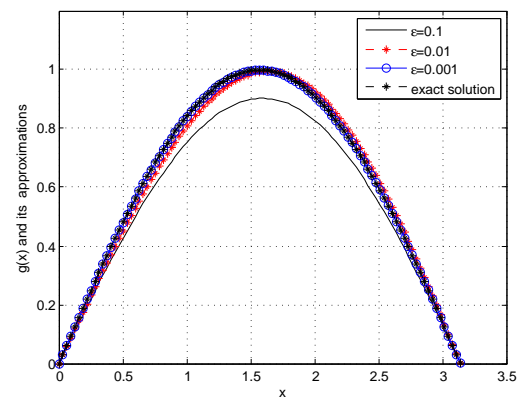

Figure 2: The comparison between the exact solution $g$ and its computed approximations $g_{\delta}^{\epsilon}$ with $M=100, N=6$ and $p=1$ for example1.

Example 2 Let $g(x)=\sin (2 x)$ and $f(x)$ be the following piecewise smooth function

$$
f(x)= \begin{cases}0, & 0 \leq x \leq \frac{\pi}{4}, \\ \frac{4}{\pi} x-1, & \frac{\pi}{4}<x \leq \frac{\pi}{2}, \\ 3-\frac{4}{\pi} x, & \frac{\pi}{2}<x \leq \frac{3 \pi}{4}, \\ 0, & \frac{3 \pi}{4}<x \leq \pi .\end{cases}
$$


In this example, we get the mesurable data $\psi_{1}(x)$ and $\psi_{2}(x)$ by solving the problem (4.8), then obtain the perturbed data according to (4.6), finally obtain the regularized solutions from (4.4) and (4.5).

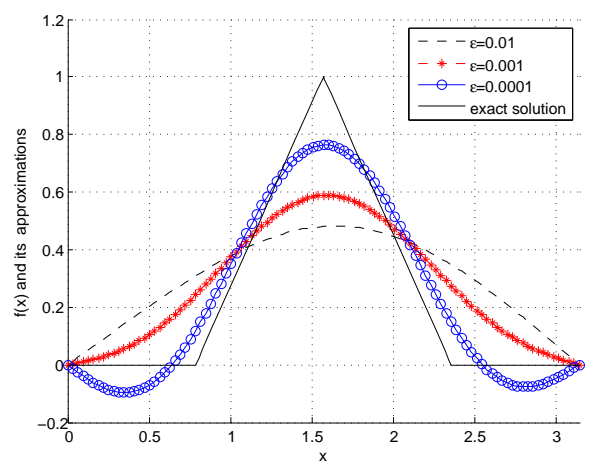

Figure 3: The comparison between the exact solution $f$ and its computed approximations $f_{\delta}^{\epsilon}$ with $M=100, N=6$ and $p=1$ for example2.

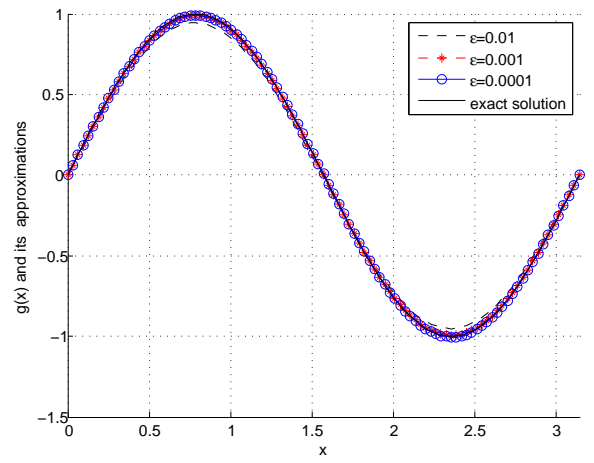

Figure 4: The comparison between the exact solution $g$ and its computed approximations $g_{\delta}^{\epsilon}$ with $M=100, N=6, p=1$ for example2.

Figure 3 (resp. Figure 4) indicates the comparison between the initial condition $f(x)$ (resp. the function $g(x)$ ) and its regularized solutions. Taking into consideration the nonsmooth and the ill posedness of the problem, the results presented in Figures 3 and 4 are reasonable. 


\section{Conclusion}

In this paper, we have proposed a modified Tikhonov regularization method to identify an unknown source term and unknown initial condition in a class of inverse boundary value problems of parabolic type, convergence results were established, and error estimates have been obtained under an a priori bound of the exact solutions. Meanwhile, a numerical example verified the efficiency and accuracy of the method.

\section{References}

[1] G. Bastay, Iterative Methods for Ill-posed Boundary Value Problems, Linkoping Studies in Science and Technology, Dissertations No. 392, Linkoping Univ. Linkoping, 1995.

[2] N. Boussetila and F. Rebbanin, Optimal regularization method for illposed Cauchy problems, Electronic Journal of Differential Equations $\mathbf{1 4 7}$ (2006), 1-15.

[3] J.R. Cannon and P.Duchateau, Structural identification of an unknown source term in a heat equation, Inverse Problems, 14 (1998), 535-551.

[4] M. Choulli and M. Yamamoto, Conditional stability in determining a heat source, Journal of Inverse and Ill-Posed Problems, 12 (2004), 233243.

[5] G.W. Clark and S.F. Oppenheimer, Quasireversibility methods for nonwell-posed problems, Electronic Journal of Differential Equations, 8 (1994), 1-9.

[6] H.W. Engl, M. Hanke and A. Neubauer, Regularization of Inverse Problem, Kluwer Academic, Boston, MA, 1996.

[7] C.L. Fu, Simplified Tikhonov and Fourier regularization methods on a general sideways parabolic equation, J. Comput. Appl. Math. 167 (2004), 449-463.

[8] JA. Goldstein, Semigroups of linear operators and applications, Oxford university, press New York. 1985.

[9] D. N. Hào, N. Van, and D. Lesnic, Regularization of parabolic equations backward in time by a non-local boundary value, IMA Journal of Applied Mathematics, vol. 75 no. 2, (2010), 291-315. 
[10] A. Hasanov, Simultaneous determination of source terms in a linear parabolic problem from the final overdetermination: Weak solution approach J. Math. Anal. Appl., 330 (2007), 766-779.

[11] Y. C. Hon and T. Takeuchi, Discretized Tikhonov regularization by reproducing kernel Hilbert space for backward heat conduction problem, Advances in Computational Mathematics, vol. 34 no. 2, (2011), 167-183.

[12] M. S. Hussein and D. Lesnic, Simultaneous determination of timedependent coefficients and heat source, International Journal for Computational Methods in Engineering Science and Mechanics, vol. 17 no. 5-6, (2016), 401-411.

[13] B. T. Johansson and D. Lesnic, A procedure for determining a spacewise dependent heat source and the initial temperature, Applicable Analysis, vol. 87 no. 3, (2008), 265276.

[14] M.M. Lavrentev, V.G. Romanov and G.P. Shishatskii, Ill-posed Problems in Mathematical Physics and Analysis, (Providence, RI: American Mathematical Society), 1986.

[15] A. Kirsch, An Introduction to the Mathematical Theory of Inverse Problems, Springer-Verlag, New York, 1996.

[16] K. Miller, Stabilized quasi-reversibility and other nearly-bestpossiblemethods for non-well-posed problems Symposium on NonWell-Posed Problems and Logarithmic Convexity (Heriot-Watt Univ. Edinburgh, 1972) (Lecture Notes in Math. vol 316) (Berlin: Springer) (1973), 161-76.

[17] A. Pazy, Semigroups of linear operators and application to partial differential equations, Springer-Verlag, 1983.

[18] S. Qiu, W. Zhang and J. Peng, Simultaneous determination of the spaceDependent source and the initial distribution in a heat equation by regularizing Fourier coefficients of the given measurements. Advances in Mathematical Physics, Vol. 2018, Article ID 8247584, 15 pages.

[19] A. M. Shahrezaee and M. Rostamian, Determination of a source termand boundary heat flux in an inverse heat equation, Journal of Information and Computing Science, vol. 8 no. 2, (2013), 103-114.

[20] N. H. Tuan and D. D. Trong, A new regularized method for two dimensional nonhomogeneous backward heat problem, Applied Mathematics and Computation, vol. 215 no. 3, (2009), 873- 880. 
[21] D.D. Trong, N.T. Long and P.N. Dinh Alain, Nonhomogeneous heat equation: identification and regularization for the inhomogeneous term, $J$. Math. Anal. Appl. 312 (2005), 93-104.

[22] N. H. Tuan and D. D. Trong, A new regularized method for two dimensional nonhomogeneous backward heat problem, Applied Mathematics and Computation, vol. 215 no. 3, (2009), 873880.

[23] T. Wei and J. C. Wang, Simultaneous determination for a spacedependent heat source and the initial data by the MFS, Engineering Analysis with Boundary Elements, vol. 36 no. 12, (2012), 1848-1855.

[24] J. Wen, M. Yamamoto, and T. Wei, Simultaneous determination of a time-dependent heat source and the initial temperature in an inverse heat conduction problem, Inverse Problems in Science and Engineering, vol. 21 no. 3, (2013), 485-499.

[25] F. Yang and C.L. Fu, A simplified Tikhonov regularization method for determining the heat source, Appl. Math. Model. 34 (2010), 3286-3299.

[26] L. Yang, Z.-C. Deng, and Y.-C. Hon, Simultaneous identification of unknown initial temperature and heat source, Dynamic Systems and Applications, vol. 25 no. 4, (2016), 583-602.

[27] Z.-X. Zhao, M. K. Banda and B.-Z. Guo, Simultaneous identification of diffusion coefficient, spacewise dependent source and initial value for onedimensional heat equation, Mathematical Methods in Applied Sciences, 40 (2017), 3552-3565.

[28] G.-H. Zheng and T. Wei, Recovering the source and initial value simultaneously in a parabolic equation, Inverse Problems, vol. 30 no. 6, Article ID 065013, (2014), 1-35.

Nabil SAOULI,

Applied Mathematics Laboratory,

University Badji Mokhtar-Annaba,

P.O.Box. 12, Annaba 23000, Algeria.

Email: nsaouli23@gmail.com

Fairouz ZOUYED,

Applied Mathematics Laboratory,

University Badji Mokhtar-Annaba,

P.O.Box. 12, Annaba 23000, Algeria

Email: fzouyed@gmail.com 